\title{
Nitrogen fertilization: Brazilian scenario and future prospects
}

\section{Opinion}

Nitrogen $(\mathrm{N})$ has been the most studied element in the last years possibly due to its dynamism, its high requirement for plants and its great possibility of loss via volatilization. Numerous studies involving $\mathrm{N}$ was published, it is important to note that the great participation of this nutrient in the works involving sources and doses and in studies with the intention of mitigating their losses. The calibration of sources and doses of $\mathrm{N}$ has been made for the most varied cultures and management methods. In this way the spectrum of the values obtained has been very varied, however it should be emphasized that the published results seem to converge towards the same direction. The clear example of this behavior is that between the years 2007 and 2010 were published about 8 articles involving grasses and nitrogen fertilization. Note that in spite of the constant addition of other variables, as a period of application, different sources, the different results obtained can be explained by the addition of these variables and by the demand of the species itself or varieties. The importance of this type of study is undeniable; especially when one thinks about the practical applications of these works, but it is noticed that in most cases these works are discussed basically comparing values with old works without the concern to explain in detail the reason of obtain these values. The viable alternative for the enrichment of this modality of work is an approach in the physiological aspects that occur in the plant during the increment of these doses, making a link between knowledge of soil fertility and mineral nutrition.

$\mathrm{N}$ is also a prominent participant in works that involve the efficiency of organic fertilizers. The use of organic residues of animal and vegetal origin has been shown as a viable alternative to supply $\mathrm{N}$ for the plants. However, due to the diversity in the chemical composition of the waste, studies have shown that in some cases the application presents efficiency when the residue is complemented by other forms of management. In general, organic vegetable residues have a lower rate of $\mathrm{N}$ release, thus requiring joint applications with other forms of fertilizers. Studies indicate that the half-life of plant residues can range from 70 to 180 days depending on the lignin content of the residue. Experiments with fertilizers with organic residues in sugarcane indicate that the highest yields are obtained with a combination of green fertilizers and inorganic $\mathrm{N}$ fertilizers in the first year, while in the second, higher yields were observed in treatments containing only the organic source. The same behavior has not been reported in studies involving organic residues from animals. The application of swine and poultry manures has increased the rate of $\mathrm{N}$ mineralization after its application, thus increasing the availability of this nutrient to the plants. However, due to their chemical composition, poultry manure can be used as fertilizer for the bean crop as long as it is supplemented with $\mathrm{N}$ and $\mathrm{P}$ minerals.
Volume 2 Issue 4 - 2018

\author{
Matheusda Silva Ferreira \\ Department of Soils, Federal University of Viçosa, Brazil
}

Correspondence: Matheusda Silva Ferreira, Department of Soils, Federal University of Viçosa, Brazil, Tel +55 92982022259 , Email matheus3ferreira@gmail.com

Received: October 31, 2017| Published: July 13, 2018

On the other hand, this limitation has not been observed in studies with swine manure that, together with the large amount of manure produced by swine, has motivated the conduction of several experiments involving this type of waste. The results indicated that the pig slurry application increases the $\mathrm{N}-\alpha$-amino, $\mathrm{N}$-ammonium and $\mathrm{N}$-amide fractions, increasing the availability of nitrogen for corn, oats, soybean and wheat plants, resulting in grain yield. Nitrogen volatilization has also been one of the main pillars studied by soil fertility. Urea is one of the main sources of nitrogen $(\mathrm{N})$ used in agriculture, but it is quite susceptible to losses due to volatilization of ammonia. The use of urease inhibitors has been reported in the literature as a way to mitigate these losses. From the practical point of view, the use of urea with NBPT inhibitor type allows its application to be preceded by up to 10 days of irrigation without damage to rice. In traditional cultivation systems the use of fertilizers with inhibitors is more effective in minimizing losses of $\mathrm{N}$ by volatilization when incorporated. It is also worth mentioning that studies involving organic compounds and $\mathrm{N}$ volatilization have shown that the ammonia emission increases with the increase of the dose of compound applied, especially during periods of higher temperature and, in the case of $\mathrm{N}_{2} \mathrm{O}$, the higher volatilization rates occur during the first 40 days, starting from the beginning of rainfall or irrigation. However, compared with other nutrients, the $\mathrm{N}$ presents a large void to be filled in scientific questions, making it necessary to carry out a larger number of studies about the N-Plant-Soil relationship.

\section{Acknowledgements}

None.

\section{Conflict of interest}

The author declares that there is no conflict of interest. 